\title{
Article
}

\section{Negative Impact of Fear of COVID-19 on Health-Related Quality of Life Was Modified by Health Literacy, eHealth Literacy, and Digital Healthy Diet Literacy: A Multi-Hospital Survey}

\author{
Minh H. Nguyen ${ }^{1}{ }^{(}$, Thu T. M. Pham ${ }^{2,3}{ }^{\circledR}$, Kien T. Nguyen ${ }^{4,5}$, Yen H. Nguyen ${ }^{6,7,8}$, Tien V. Tran ${ }^{9,10}$, \\ Binh N. Do ${ }^{9,11}{ }^{1}$, Hung K. Dao ${ }^{12}$, Huu C. Nguyen ${ }^{13,14}$, Ngoc T. Do ${ }^{15}$, Tung H. Ha ${ }^{16}$, Dung T. Phan ${ }^{17,18}$, \\ Khue M. Pham ${ }^{2,19}{ }^{(0)}$, Linh V. Pham ${ }^{20,21}{ }^{(0)}$, Phuoc B. Nguyen ${ }^{22}$, Hoai T. T. Nguyen ${ }^{23}$, Thinh V. Do ${ }^{24}$, Dung T. Ha ${ }^{25}$, \\ Hung Q. Nguyen ${ }^{26}$, Huong T. M. Ngo ${ }^{27}$, Manh V. Trinh ${ }^{28}$, Thuy T. T. Mai ${ }^{29}$, Nhan P. T. Nguyen ${ }^{30}$, Anh L. Tra ${ }^{31}$, \\ Thao T. P. Nguyen ${ }^{32,33}$, Kien T. Nguyen ${ }^{34}$, Chyi-Huey Bai ${ }^{1,3,35, *(\mathbb{D})}$ and Tuyen Van Duong ${ }^{36, *(1)}$
}

check for
updates

Citation: Nguyen, M.H.; Pham, T.T.M.; Nguyen, K.T.; Nguyen, Y.H.; Tran, T.V.; Do, B.N.; Dao, H.K.; Nguyen, H.C.; Do, N.T.; Ha, T.H.; et al. Negative Impact of Fear of COVID-19 on Health-Related Quality of Life Was Modified by Health Literacy, eHealth Literacy, and Digital Healthy Diet Literacy: A Multi-Hospital Survey. Int. J. Environ. Res. Public Health 2021, 18, 4929. https://doi.org/10.3390/ ijerph18094929

Academic Editors: Mariusz Duplaga and Tetine Sentell

Received: 6 April 2021

Accepted: 28 April 2021

Published: 6 May 2021

Publisher's Note: MDPI stays neutral with regard to jurisdictional claims in published maps and institutional affiliations.

Copyright: (c) 2021 by the authors. Licensee MDPI, Basel, Switzerland. This article is an open access article distributed under the terms and conditions of the Creative Commons Attribution (CC BY) license (https:/ / creativecommons.org/licenses/by/ $4.0 /)$.
1 International Ph.D. Program in Medicine, College of Medicine, Taipei Medical University, Taipei 110-31, Taiwan; d142108015@tmu.edu.tw

2 Faculty of Public Health, Hai Phong University of Medicine and Pharmacy, Hai Phong 042-12, Vietnam; phamminhthu.ytcc@gmail.com (T.T.M.P.); pmkhue@hpmu.edu.vn (K.M.P.)

3 School of Public Health, College of Public Health, Taipei Medical University, Taipei 110-31, Taiwan

4 President Office, Can Tho University of Medicine and Pharmacy, Can Tho 941-17, Vietnam; ntkien@ctump.edu.vn

5 Department of Physiology, Faculty of Medicine, Can Tho University of Medicine and Pharmacy, Can Tho 941-17, Vietnam

6 Department of Pharmacology and Clinical Pharmacy, Can Tho University of Medicine and Pharmacy, Can Tho 941-17, Vietnam; nhyen@ctump.edu.vn

Department of Pharmacy, Can Tho University of Medicine and Pharmacy Hospital, Can Tho 941-17, Vietnam

8 Ph.D. Program in School of Pharmacy, College of Pharmacy, Taipei Medical University, Taipei 110-31, Taiwan

9 Department of Infectious Diseases, Vietnam Military Medical University, Hanoi 121-08, Vietnam; tientv@vmmu.edu.vn (T.V.T.); nhubinh.do@vmmu.edu.vn (B.N.D.)

10 Director Office, Military Hospital 103, Hanoi 121-08, Vietnam

11 Division of Military Science, Military Hospital 103, Hanoi 121-08, Vietnam

12 Director Office, Bac Ninh Obstetrics and Pediatrics Hospital, Bac Ninh 161-23, Vietnam; daokhachung2000@yahoo.com

13 Director Office, E Hospital, Hanoi 113-08, Vietnam; bacsyhuu@gmail.com

14 Department of Thoracic and Cardiovascular Surgery, E Hospital, Hanoi 113-08, Vietnam

15 Nursing Office, E Hospital, Hanoi 113-08, Vietnam; dothingocbve@gmail.com

16 Director Office, General Hospital of Agricultural, Hanoi 125-16, Vietnam; hahuutung.200564@gmail.com

17 Faculty of Nursing, Hanoi University of Business and Technology, Hanoi 116-22, Vietnam; phanthidzungvd@gmail.com

18 Nursing Office, Thien An Obstetrics and Gynecology Hospital, Hanoi 112-06, Vietnam

19 President Office, Hai Phong University of Medicine and Pharmacy, Hai Phong 042-12, Vietnam

20 Department of Pulmonary \& Cardiovascular Diseases, Hai Phong University of Medicine and Pharmacy Hospital, Hai Phong 042-12, Vietnam; pvlinh@hpmu.edu.vn

21 Director Office, Hai Phong University of Medicine and Pharmacy Hospital, Hai Phong 042-12, Vietnam

22 Director Office, Kien An Hospital, Hai Phong 046-09, Vietnam; nguyenbatuankiet@gmail.com

23 Training and Direction of Healthcare Activity Center, Kien An Hospital, Hai Phong 046-09, Vietnam; hoaibvka@gmail.com

24 Director Office, Bai Chay Hospital, Quang Ninh 011-21, Vietnam; dovanthinhhscc@gmail.com

25 Nursing Office, Bai Chay Hospital, Quang Ninh 011-21, Vietnam; hadungbvbc@gmail.com

26 Director Office, Quang Ninh Obstetrics and Pediatrics Hospital, Quang Ninh 011-24, Vietnam; bshungbvqn@gmail.com

27 Nursing Office, Quang Ninh Obstetric and Pediatric Hospital, Quang Ninh 011-24, Vietnam; ngomaihuong.hl@gmail.com

28 Director Office, Quang Ninh General Hospital, Quang Ninh 011-08, Vietnam; trinhmanhqnsyt@gmail.com

29 Nursing Office, Quang Ninh General Hospital, Quang Ninh 011-08, Vietnam; maithanhthuy68@gmail.com

30 General Planning Department, Da Nang Oncology Hospital, Da Nang 506-06, Vietnam; bsnhanbvub@gmail.com

31 Department of Physiotherapy and Rehabilitation, Da Nang University of Medical Technology and Pharmacy, Da Nang 502-06, Vietnam; hanitra2630@gmail.com 
32 Health Management Training Institute, University of Medicine and Pharmacy, Hue University, Thua Thien Hue 491-20, Vietnam; nguyenthiphuongthao@hueuni.edu.vn

33 Department of Health Economics, Corvinus University of Budapest, 1093 Budapest, Hungary

34 Department of Health Education, Faculty of Social Sciences, Behavior and Health Education, Hanoi University of Public Health, Hanoi 119-10, Vietnam; ntk1@huph.edu.vn

35 Department of Public Health, College of Medicine, Taipei Medical University, Taipei 110-31, Taiwan

36 School of Nutrition and Health Sciences, Taipei Medical University, Taipei 110-31, Taiwan

* Correspondence: baich@tmu.edu.tw (C.-H.B.); tvduong@tmu.edu.tw (T.V.D.); Tel.: +886-2-2736-1661 (ext. 6510) (C.-H.B.); +886-2-2736-1661 (ext. 6545) (T.V.D.)

\begin{abstract}
Background: The COVID-19 pandemic has been disseminating fear in the community, which has affected people's quality of life, especially those with health problems. Health literacy (HL), eHealth literacy (eHEAL), and digital healthy diet literacy (DDL) may have potential impacts on containing the pandemic and its consequences. This study aimed to examine the association between the fear of COVID-19 scale (FCoV-19S) and the health-related quality of life (HRQoL), and to examine the effect modification by HL, eHEAL, and DDL on this association. Methods: A crosssectional study was conducted in 11 hospitals across Vietnam from 7 April to 31 May 2020. Data were collected on 4348 outpatients, including demographic characteristics, HL, eHEAL, DDL, FCoV-19S, and HRQoL. Multiple linear regression and interaction models were used to explore associations. Results: Patients with higher FCoV-19S scores had lower HRQoL scores (unstandardized coefficient, $\mathrm{B}=-0.78, p<0.001)$. HL $(\mathrm{B}=0.20, p<0.001)$, eHEAL $(\mathrm{B}=0.24, p<0.001)$, and DDL $(\mathrm{B}=0.20$, $p<0.001)$ were positively associated with higher HRQoL scores. The negative impact of FCoV-19S on HRQoL was significantly attenuated by higher eHEAL score groups (from one standard deviation (SD) below the mean, $\mathrm{B}=-0.93, p<0.001$; to the mean, $\mathrm{B}=-0.85, p<0.001$; and one SD above the mean, $\mathrm{B}=-0.77, p<0.001$ ); and by higher DDL score groups (from one SD below the mean, $\mathrm{B}=-0.92, p<0.001$; to the mean, $\mathrm{B}=-0.82, p<0.001$; and one $\mathrm{SD}$ above the mean, $\mathrm{B}=-0.72$, $p<0.001)$. Conclusions: eHealth literacy and digital healthy diet literacy could help to protect patients' health-related quality of life from the negative impact of the fear of COVID-19 during the pandemic.
\end{abstract}

Keywords: fear of COVID-19; health-related quality of life; health literacy; eHealth literacy; digital healthy diet literacy

\title{
1. Introduction
}

The COVID-19 pandemic has been placing unprecedented challenges and burdens on the economic, health, and political systems of the affected countries [1-3]. The numbers of new infections and deaths are increasing, and there is no sign of control [4]. Therefore, the COVID-19 pandemic has been significantly affecting people's health and well-being around the world.

Preventive measures have been implemented worldwide to control COVID-19 transmissions such as lockdowns, social distancing, mask-wearing, and handwashing [5]. However, these measures have also caused a wide range of negative consequences such as a lack of social connection, mental health problems, and lifestyle changes [6,7]. Thus, people's psychological and physical health have seriously been affected, resulting in a deterioration in the health-related quality of life (HRQoL). Therefore, it is crucial to determine the risk and protective factors affecting the health-related quality of life during a pandemic to develop early and effective interventions to improve the HRQoL.

The COVID-19 pandemic is still rapidly spreading with record numbers of new infections and deaths, leading to an increased fear of virus transmission in the community. Fear may cause adverse effects such as lifestyle changes, delays in healthcare access, and mental health problems such as depression or even suicide [8,9]. Thus, it can affect people's quality of life, especially high-risk groups such as the elderly and people with health problems $[10,11]$. 
During the time of social distancing and limited connection with others, people spent more time interacting on social media. Social media has become a popular means for communication, entertainment, and accessing health information $[12,13]$. However, social media has also been rapidly spreading false and inaccurate information about COVID19, which may raise concerns and fears in the public [14]. eHealth literacy (eHEAL) is the capacity to seek, find, understand, and appraise basic health information from Web-based sources and to apply the knowledge acquired to solve health problems [15]. Therefore, eHealth literacy and health literacy (HL) have potential roles in dealing with misinformation and containing the spread of COVID-19 in the community [16,17]. Besides, improving HL and eHEAL could help people engage in active behaviors such as healthy diets, physical activities, and adherence to preventive measures during the pandemic [18], which could further improve the health status and well-being [19].

Healthy diets have shown beneficial effects on the enhancement of immune systems and health outcomes during the COVID-19 pandemic [20-22]. Moreover, a wellbalanced and adequate diet can improve the immune response to viral infections (e.g., COVID-19) [23], and reduces the risk of chronic diseases [24,25]. In a recent study, healthy dietary intake was a protective factor that could reduce the risk of depression during the COVID-19 lockdown period [26]. Thus, eating a healthy diet is crucial to help people stay fit, which improves their quality of life during the pandemic. However, unbalanced eating habits are rising worldwide [27,28], including in Vietnam [29]. During the pandemic, governments have implemented preventive measures (e.g., lockdowns and social distancing), which has negatively impacted people's mental health and living habits, including diet and eating behaviors. Therefore, it is essential to improve the knowledge about healthy and proper diets during the COVID-19 crisis. Digital healthy diet literacy (DDL) is the ability to seek, understand, appraise, and apply healthy diet information from electronic sources to improve healthy eating habits [30]. A previous study conducted in Vietnam indicated that DDL was positively associated with healthier eating habits [30]. Hence, by improving healthy diets, DDL may have a potential effect in enhancing the quality of life during a pandemic. However, to date, no studies have evaluated that relationship. Therefore, assessing the relationship between DDL and HRQoL is critical to providing timely suggestions for public health interventions and future studies.

This study was conducted to explore the association of the fear of COVID-19 (FCoV19S), HL, eHEAL, and DDL with the health-related quality of life (HRQoL), and to examine the effect modification by HL, eHEAL, and DDL on the association between FCoV-19S and HRQoL among outpatients in 11 hospitals across Vietnam.

\section{Materials and Methods}

\subsection{Study Design and Settings}

We conducted a cross-sectional study between 7 April and 31 May 2020 at 11 hospitals in Vietnam, including nine hospitals in the Northern area, one hospital in the Central area, and one hospital in the Southern area.

The study was reviewed and approved by the Institutional Ethical Review Committee of Hanoi University of Public Health, Vietnam (IRB No. 133/2020/YTCC-HD3).

\subsection{Study Sample and Data Collection}

During a pandemic, all resources and budgets in hospitals are re-arranged for epidemic prevention. In addition, it has been urgent to provide timely evidence for interventions to improve the quality of life for the outpatient population during a pandemic, so we used the convenience sampling method, which is a rapid method and minimizes financial and human burdens. Patients who visited the outpatient departments (OPD) at the selected hospitals were recruited into the study. Eligible participants were those aged between 18 and 85 years, who were able to read and write Vietnamese, and who agreed to join the survey. Patients with any emergency conditions (e.g., stroke, respiratory failure, and serious injuries), dementia, psychotic disorder, or blindness were excluded. Finally, the overall 
sample of 4348 respondents was collected and analyzed in our study. The distribution of recruited participants from each hospital is presented in Table 1.

Table 1. Study sample at 11 hospitals across Vietnam.

\begin{tabular}{llc}
\hline \multicolumn{1}{c}{ Geographic Location } & \multicolumn{1}{c}{ Hospitals } & \multicolumn{1}{c}{$\begin{array}{c}\text { Studied } \\
\text { Participants }\end{array}$} \\
\hline $\begin{array}{l}\text { Northern area } \\
\text { Ha Noi city }\end{array}$ & \multicolumn{1}{c}{ 1. Military Hospital 103 } & 183 \\
& 2. E Hospital & 300 \\
3. General Hospital of Agricultural & \\
Hai Phong city & 4. Hai Phong University of Medicine and & 490 \\
& Pharmacy Hospital & 492 \\
Quang Ninh province & 5. Kien An Hospital & 364 \\
& 6. Bai Chay Hospital & 280 \\
7. Quang Ninh Obstetrics and Pediatrics & 309 \\
Bac Ninh city & Hospital Quang Ninh General Hospital & 500 \\
Central area & 9. Bac Ninh Obstetrics and Pediatrics Hospital \\
Da Nang city & & \\
Southern area & 10. Da Nang Oncology Hospital & 421 \\
Can Tho city &
\end{tabular}

At each selected hospital, research assistants (medical staff and medical students) were trained in 2 sessions: (1) data collection; (2) infection control (e.g., mask-wearing, washing hands, and physical distancing) using guidelines of the World Health Organization [31].

The research assistants contacted and consecutively invited outpatients who presented at the OPDs to join the survey. Patients voluntarily participated and signed the informed consent form before conducting the investigation. We used a self-administered questionnaire to collect the data. People who visited hospitals could either pick a printed self-administered questionnaire or scan the QR code using their smartphone to link to the online questionnaire. In most of the hospitals, the participants used printed selfadministered questionnaires to complete the survey. During the survey period, research assistants constantly supervised and assisted the participants in completing the survey. It took 15-20 min to complete each survey. Finally, the data were cleaned and coded for analysis.

\subsection{Instruments and Measurements}

\subsubsection{Health-Related Quality of Life}

The health-related quality of life (HRQoL) was assessed with the short form (SF36) [32], which is widely used in Vietnam studies [33,34]. The self-administered SF-36 consisted of 36 questions evaluating the quality of life with 8 health aspects (e.g., vitality, physical function, bodily pain, general health, physical role function, emotional role function, and mental health). The scoring method was provided in the user guidelines [35]. The overall score varied between 0 and 100, with a higher score indicating a better HRQoL. 


\subsubsection{Fear of COVID-19}

The fear of COVID-19 was evaluated using a 7-item fear of COVID-19 scale (FCoV19S), which has been developed and validated in Iran [36] and in Vietnam [37], with satisfactory reliability and validity. The Cronbach's alpha of FCoV-19S in this study was 0.92 . Respondents rated their perception about the susceptibility to COVID-19 infectability on a 5 -point Likert scale from 1 (strongly agree) to 5 (strongly disagree). The responses were summed up, and the total score was between 7 and 35, with a higher score representing a higher level of fear of COVID-19.

\subsubsection{Health Literacy and Digital Healthy Diet Literacy}

Health literacy (HL) was measured with a short-form health literacy questionnaire (HLS-SF12), which was widely used for studies in Asia [38] and in Vietnam [34,39-41]. The Cronbach's alpha of HLS-SF12 in the current study was 0.95.

Digital healthy diet literacy (DDL) was measured using a DDL-4 questionnaire. This questionnaire has been developed and validated in Vietnam, and the reliability and validity were satisfactory [30]. The Cronbach's alpha of DDL-4 was 0.96 in this study.

The HLS-SF12 and DDL-4 questionnaires consisted of 12 and 4 items, respectively. Patients responded to the questionnaires on the 4-point Likert scale regarding the difficulty in conducting each item ranging from 1 (very difficult) to 4 (very easy). The HL and DDL scores were transformed into a unified index between 0 and 50, with a higher score indicating a better HL or DDL. The formula has been described in a previous study [30].

\subsection{4. eHealth Literacy}

The participants' eHealth literacies were measured with an 8-item eHealth literacy scale (eHEALS), which has been validated and used in Vietnam, and the reliability and validity were satisfactory [18]. In our study, the Cronbach's alpha of eHEALS was 0.96. Respondents rated their degree of agreement with 8 items about their experiences in healthrelated information processing on the internet on a 5-point Likert scale ranging from 1 (strongly disagree) to 5 (strongly agree). The total score was between 8 and 40, with a higher eHEALS score indicating a better eHealth literacy.

\subsubsection{Participants' Characteristics}

The patients' demographic information was collected, including age (years), sex (men vs. women), marital status (never married vs. ever married), education degree (secondary school or below, high school, or college/university or higher), occupational status (unemployed/dependents vs. employed), ability to pay for medication (very difficult to very easy), and social status (patients self-assessed their position in the society in terms of education, occupation, and income at 3 degrees of low, middle, and high). Participants reported their body weight $(\mathrm{kg})$ and height $(\mathrm{cm})$. Body mass index $\left(\mathrm{BMI}, \mathrm{kg} / \mathrm{m}^{2}\right)$ was calculated and classified into 2 groups (normal weight (BMI < 25.0) vs. overweight/obese $(\mathrm{BMI} \geq 25.0)$ ).

Respondents were screened for the suspected COVID-19 symptoms (S-COVID-19-S) at the time of the survey, including common symptoms (fever, cough, and dyspnea) and less common symptoms (myalgia, fatigue, sputum production, confusion, headache, sore throat, rhinorrhea, chest pain, hemoptysis, diarrhea, and nausea/vomiting) [42]. Participants were classified as having suspected COVID-19 symptoms if they had any of these symptoms. Respondents self-reported their comorbidities using the Charlson Comorbidity Index items [43]. We classified the participants into 2 groups (with vs. without comorbidity).

\subsubsection{Lifestyle Changes}

Patients provided their information about current lifestyle behaviors in comparison to those before the epidemic. Smoking, drinking, and physical activity were assessed on a five-response scale: never, stopped, less, unchanged, and more. Eating behavior was reported on a three-response scale: healthy, unchanged, healthier. According to WHO 
recommendations, to stay fit during the pandemic, people should maintain or improve their living habits, such as staying physically active, eating a healthy diet, quitting tobacco, and avoiding alcohol [44]. The "unchanged or healthier" diet and "unchanged or more" physical activity were considered as positive behaviors, and "unchanged or more" smoking or drinking were considered as negative behaviors. Therefore, patients' responses were categorized into two groups: "never/stopped/less" vs. "unchanged or more" for smoking, drinking, and physical activity, and "less healthy" vs. "unchanged or healthier" for healthy eating.

\subsection{Statistical Analysis}

First, the distribution of studied variables was presented with a number (n), percentage $(\%)$, mean, and standard deviation (SD) appropriately. Next, the t-test or one-way ANOVA test was used to explore group differences in HRQoL scores. Thirdly, the associations of FCoV-19S, HL, eHEALS, and DDL with HRQoL were analyzed in different individual models using simple and adjusted linear regression analysis. In addition to age and gender, other factors correlated with HRQoL in the bivariate analysis $(p<0.20)$ were adjusted in multivariate analysis. To eliminate the multicollinearity, we analyzed correlations between adjusted factors using Spearman's correlation. If two factors showed a moderate correlation (rho $\geq 0.30$ ), one representative factor was put into the adjusted models. These results are given in Tables S1-S3 in Supplementary Materials. Finally, we examined the modification effects of HL, eHEALS, and DDL on the association between FCoV-19S and HRQoL using interaction models. If the interaction term was statistically significant, we conducted the simple slope analysis to visualize the interaction effect. To perform simple slope analysis, three variables, FCoV-19S, eHEALS, and DDL, were standardized into Z-scores with a mean of zero and a standard deviation of one. The plots were drawn by evaluating the values of HRQoL for three values of both the continuous independent variable FCoV-19S and continuous moderator variables (eHEALS or DDL), including $Z=1$ (one standard deviation above the mean), $Z=0$ (the mean), and $Z=-1$ (one standard deviation below the mean), and creating three lines to indicate the effect of FCoV-19S on the HRQoL at the three values of eHEALS or DDL. The $p$-value $<0.05$ was defined as significant. We used the IBM SPSS Version 20.0 for data analysis (IBM Corp, Armonk, NY, USA).

\section{Results}

\subsection{Demographic Characteristics}

The average age of the sample was 42.8 years. The mean scores of HL, eHEALS, DDL, and FCoV-19S were 26.5, 27.9, 25.9, and 20.6, respectively. Of 4348 respondents, $62 \%$ were women, $82.2 \%$ ever married, $49.3 \%$ had college/university or higher degrees, $89.1 \%$ were employed, $37.5 \%$ felt it was easy to pay for treatments, $12.6 \%$ were overweight or obese, and $40.3 \%$ had suspected COVID-19 symptoms. In terms of the changes in health-related behaviors as compared to those before the pandemic, the proportion of participants with "unchanged or healthier" eating behaviors was $92.5 \%$, while the figures for "unchanged or more" smoking, "unchanged or more" drinking alcohol, and "unchanged or more" physical activity were $8.1 \%, 7.4 \%, 42.2 \%$, respectively (Table 2 ). 
Table 2. Characteristics and health-related quality of life among outpatients $(\mathrm{N}=4348)$.

\begin{tabular}{|c|c|c|c|}
\hline \multirow{2}{*}{ Variables } & \multirow{2}{*}{$\begin{array}{c}\text { Total } \\
(\mathrm{N}=4348)\end{array}$} & \multicolumn{2}{|l|}{ HRQoL } \\
\hline & & Mean (SD) & $p^{*}$ \\
\hline Age (years), mean (SD) & $42.8(16.7)$ & & \\
\hline Age groups & & & $<0.001$ \\
\hline$<60$ & $3412(78.5)$ & $65.8(17.6)$ & \\
\hline$\geq 60$ & $936(21.5)$ & $53.8(15.6)$ & \\
\hline Gender & & & 0.454 \\
\hline Women & $2694(62.0)$ & $63.0(18.1)$ & \\
\hline Men & $1654(38.0)$ & $63.5(17.5)$ & \\
\hline Marital status & & & $<0.001$ \\
\hline Never married & $772(17.8)$ & $69.7(15.6)$ & \\
\hline Ever married & $3560(82.2)$ & $61.8(18.1)$ & \\
\hline Education attainment & & & $<0.001$ \\
\hline Secondary school or below & 1007 (23.2) & $58.2(18.3)$ & \\
\hline High school & $1196(27.5)$ & $61.1(18.2)$ & \\
\hline College/university or higher & $2139(49.3)$ & $66.8(16.7)$ & \\
\hline Occupation & & & $<0.001$ \\
\hline Unemployed & $474(10.9)$ & $56.5(19.5)$ & \\
\hline Employed & $3874(89.1)$ & $64.0(17.5)$ & \\
\hline Ability to pay for treatments & & & $<0.001$ \\
\hline Very or fairly difficult & $2712(62.5)$ & $60.9(17.7)$ & \\
\hline Very or fairly easy & $1626(37.5)$ & $67.1(17.5)$ & \\
\hline Social status & & & $<0.001$ \\
\hline Low & $921(21.2)$ & $59.6(20.6)$ & \\
\hline Middle or high & $3419(78.8)$ & $64.2(16.9)$ & \\
\hline $\mathrm{BMI}, \mathrm{kg} / \mathrm{m}^{2}$ & & & 0.156 \\
\hline Normal weight $(\mathrm{BMI}<25.0)$ & $3791(87.4)$ & $63.3(18.0)$ & \\
\hline Overweight/obese (BMI $\geq 25.0)$ & $546(12.6)$ & $62.2(17.2)$ & \\
\hline Suspected COVID-19 symptoms ** & & & $<0.001$ \\
\hline No & $2595(59.7)$ & $65.5(17.2)$ & \\
\hline Yes & $1753(40.3)$ & $59.7(18.3)$ & \\
\hline Comorbidity & & & $<0.001$ \\
\hline No & $3094(71.2)$ & $64.6(17.8)$ & \\
\hline Yes & $1254(28.8)$ & $59.6(17.6)$ & \\
\hline Smoking tobacco $* * *$ & & & 0.329 \\
\hline Never, stopped, or smoke less & 3994 (91.9) & $63.1(18.0)$ & \\
\hline Unchanged or smoke more & $354(8.1)$ & $64.1(16.4)$ & \\
\hline Drinking alcohol *** & & & 0.918 \\
\hline Never, stopped, or drink less & $4015(92.6)$ & $63.2(18.1)$ & \\
\hline Unchanged or drink more & $321(7.4)$ & $63.3(15.6)$ & \\
\hline Physical activity *** & & & $<0.001$ \\
\hline Never, stopped, or exercise less & $2515(57.8)$ & $58.5(17.5)$ & \\
\hline Unchanged or exercise more & $1833(42.2)$ & $69.7(16.3)$ & \\
\hline Healthy eating ${ }^{* * *}$ & & & $<0.001$ \\
\hline Less healthy & $325(7.5)$ & $55.6(12.8)$ & \\
\hline Unchanged or healthier & $4002(92.5)$ & $63.8(18.1)$ & \\
\hline Health literacy, mean (SD) & $26.5(10.5)$ & & \\
\hline eHealth Literacy Scale, mean (SD) & $27.9(6.9)$ & & \\
\hline Digital healthy diet literacy, mean (SD) & $25.9(12.2)$ & & \\
\hline Fear of COVID-19 Scale, mean (SD) & $20.6(5.4)$ & & \\
\hline
\end{tabular}




\subsection{Associations of HL, eHEALS, DDL, and FCoV-19S with HRQoL}

In the bivariate models, age, marital status, education, occupation, ability to pay for medications, social status, BMI, comorbidity, S-COVID-19-S, physical activity, and healthy eating were associated with HRQoL at $p<0.20$ (Table S1 in Supplementary Materials). To avoid multicollinearity, the correlations among the confounders were examined. Moderate correlations were found between age and education $(r h o=-0.34)$; between the ability to pay for medications and social status $(r h o=0.30)$; between comorbidity with S-COVID-19-S $(r h o=0.50)$, HL $(r h o=-0.38)$, eHEALS $(r h o=-0.38)$, and DDL $(r h o=-0.37)$; between SCOVID-19-S and HL ( $r h o=-0.34)$, eHEALS $(r h o=-0.35)$, and DDL $(r h o=-0.31)$ (Table S2 in Supplementary Materials). Therefore, age, gender, marital status, occupation, social status, BMI, physical activity, and healthy eating were put into the adjusted models. After adjusting for confounders, multiple linear regression models showed that patients with a high level of FCoV-19S had a lower HRQoL score (unstandardized regression coefficient, $\mathrm{B}=-0.78,95 \% \mathrm{CI}-0.87,-0.70, p<0.001)$. eHEALS $(\mathrm{B}=0.24,95 \%$ CI $0.17,0.32, p<0.001)$ and HL ( $\mathrm{B}=0.20,95 \% \mathrm{CI} 0.15,0.25, p<0.001)$ were positively associated with HRQoL. A higher DDL score was found to be associated with a higher HRQoL ( $\mathrm{B}=0.18,95 \% \mathrm{CI} 0.14$, $0.22, p<0.001$; Table 3).

Table 3. Associations of fear of COVID-19, health literacy, eHealth literacy, and digital healthy diet literacy with healthrelated quality of life using multiple linear regression models $(\mathrm{N}=4348)$.

\begin{tabular}{|c|c|c|c|c|}
\hline \multirow{3}{*}{ Variables } & \multicolumn{4}{|c|}{ HRQoL } \\
\hline & \multicolumn{2}{|c|}{ Model 1** } & \multicolumn{2}{|c|}{ Model $2 * * *$} \\
\hline & B $(95 \%$ CI $)$ & $p$ & B $(95 \%$ CI) & $p$ \\
\hline Fear of COVID-19 Scale, 1-score increment * & $-0.83(-0.93,-0.74)$ & $<0.001$ & $-0.78(-0.87,-0.70)$ & $<0.001$ \\
\hline Health literacy, 1-score increment * & $0.41(0.36,0.46)$ & $<0.001$ & $0.20(0.15,0.25)$ & $<0.001$ \\
\hline eHealth Literacy Scale, 1-score increment * & $0.50(0.42,0.57)$ & $<0.001$ & $0.24(0.17,0.32)$ & $<0.001$ \\
\hline Digital healthy diet literacy, 1-score increment * & $0.38(0.33,0.42)$ & $<0.001$ & $0.18(0.14,0.22)$ & $<0.001$ \\
\hline
\end{tabular}

Abbreviation: HRQoL, health-related quality of life; B, unstandardized regression coefficient; CI, confidence interval; COVID-19, coronavirus disease 2019. * The associations of the Fear of COVID-19 Scale, health literacy, eHealth literacy, and digital healthy diet literacy with health-related quality of life were analyzed in separate individual models. ${ }^{* *}$ Model 1: Simple linear regression model. ${ }^{* * *}$ Model 2: Adjusted for age, gender, marital status, occupation, social status, BMI, physical activity, and healthy eating.

\subsection{Effect Modification by eHEALS and DDL on the Association between FCoV-19S and HRQoL}

Table 4 shows the interaction model between eHealth literacy and the fear of COVID19. Compared to patients with the lowest FCoV-19S score and eHEALS score, those with the lowest eHEALS score and 1 FCoV-19S-score increment had lower HRQoL scores $(\mathrm{B}=-1.18,95 \% \mathrm{CI}-1.51,-0.85, p<0.001)$, while those with 1 eHEALS-score increment and 1 FCoV-19S-score increment had higher HRQoL scores $(B=0.01,95 \% C I$ 0.01, 0.02, $p=0.034$ ) (Table 4). Simple slope analysis showed that when eHEALS is higher, the impact of FCoV-19S on HRQoL becomes weaker. This impact was attenuated by higher eHEALS groups from the value of $1 \mathrm{SD}$ below the mean $(\mathrm{B}=-0.93,95 \% \mathrm{CI}-1.06,-0.81, p<0.001)$ to the mean $(\mathrm{B}=-0.85,95 \% \mathrm{CI}-0.94,-0.76, p<0.001)$, and the value of $1 \mathrm{SD}$ above the mean $(\mathrm{B}=-0.77,95 \% \mathrm{CI}-0.88,-0.66, p<0.001)$. The model is visualized in Figure 1 .

In the interaction model between digital healthy diet literacy and fear of COVID-19, as compared to patients with the lowest FCoV-19S score and DDL score, those with the lowest DDL score and $1 \mathrm{FCoV}-19 \mathrm{~S}$-score increment had lower HRQoL scores $(\mathrm{B}=-1.02,95 \% \mathrm{CI}$ $-1.22,-0.82, p<0.001)$, while those with 1 DDL-score increment and 1 FCoV-19S-score increment had higher HRQoL scores $(B=0.01,95 \%$ CI 0.00, $0.02, p=0.016$ ) (Table 4). Simple slope analysis showed that when DDL is higher, the association between FCoV-19S and HRQoL becomes weaker. The impacts of FCoV-19S on HRQoL were lowered by higher DDL score groups from the value of $1 \mathrm{SD}$ below the mean $(\mathrm{B}=-0.92,95 \% \mathrm{CI}-1.04,-0.79$, $p<0.001)$, to the mean $(B=-0.82,95 \% \mathrm{CI}-0.91,-0.73, p<0.001)$, and the value of $1 \mathrm{SD}$ 
above the mean $(\mathrm{B}=-0.72,95 \% \mathrm{CI}-0.83,-0.63, p<0.001)$. The model is visualized in Figure 2 .

Table 4. Interactions of the fear of COVID-19 with health literacy, eHealth literacy, and digital healthy diet literacy on health-related quality of life $(n=4348)$.

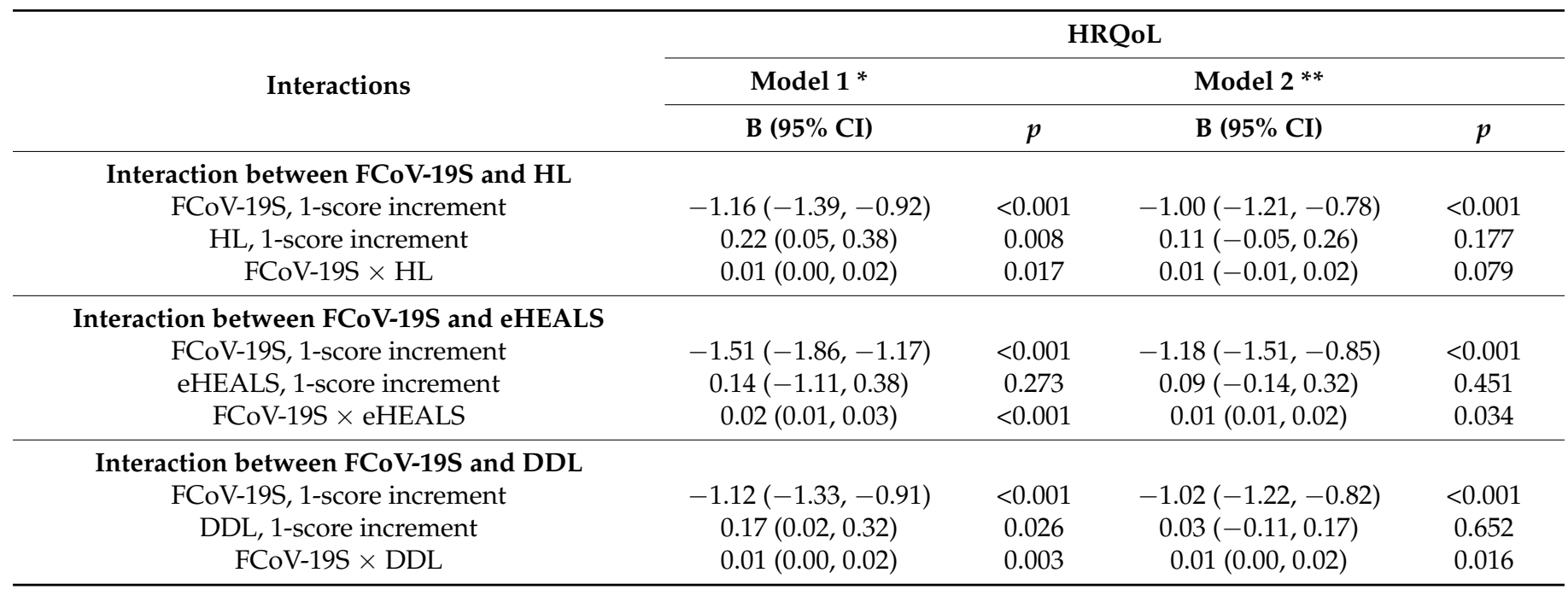

Abbreviations: HRQoL, health-related quality of life; B, unstandardized regression coefficient; CI, confidence interval; FCoV-19S, fear of coronavirus disease 2019 Scale; HL, health literacy; eHEALS, eHealth literacy; DDL, digital healthy diet literacy. ${ }^{*}$ Model 1: Simple linear regression model. ${ }^{* *}$ Model 2: Adjusted for age, gender, marital status, occupation, social status, BMI, physical activity, and healthy eating.

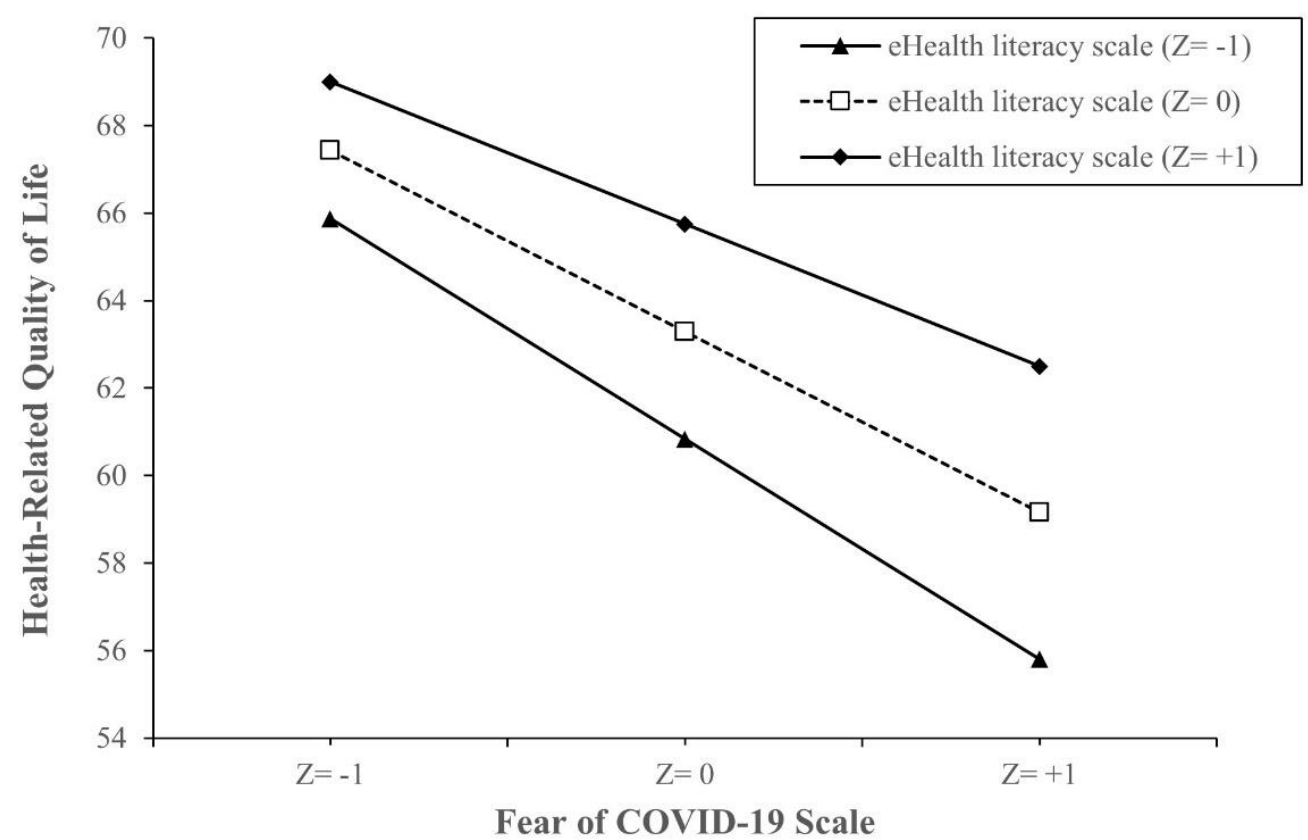

Figure 1. Simple slope plot of interaction between eHealth literacy and fear of COVID-19 on healthrelated quality of life $(N=4348)$. Note: $Z=-1$, one $S D$ below the mean; $Z=0$, the mean; $Z=+1,1 S D$ above the mean. Z-score is the number of SD above or below the mean. Abbreviations: SD, standard deviation.

The result did not show the significant effect modification by HL on the association between FCoV-19S and HRQoL in the multivariate model (Table 4). 


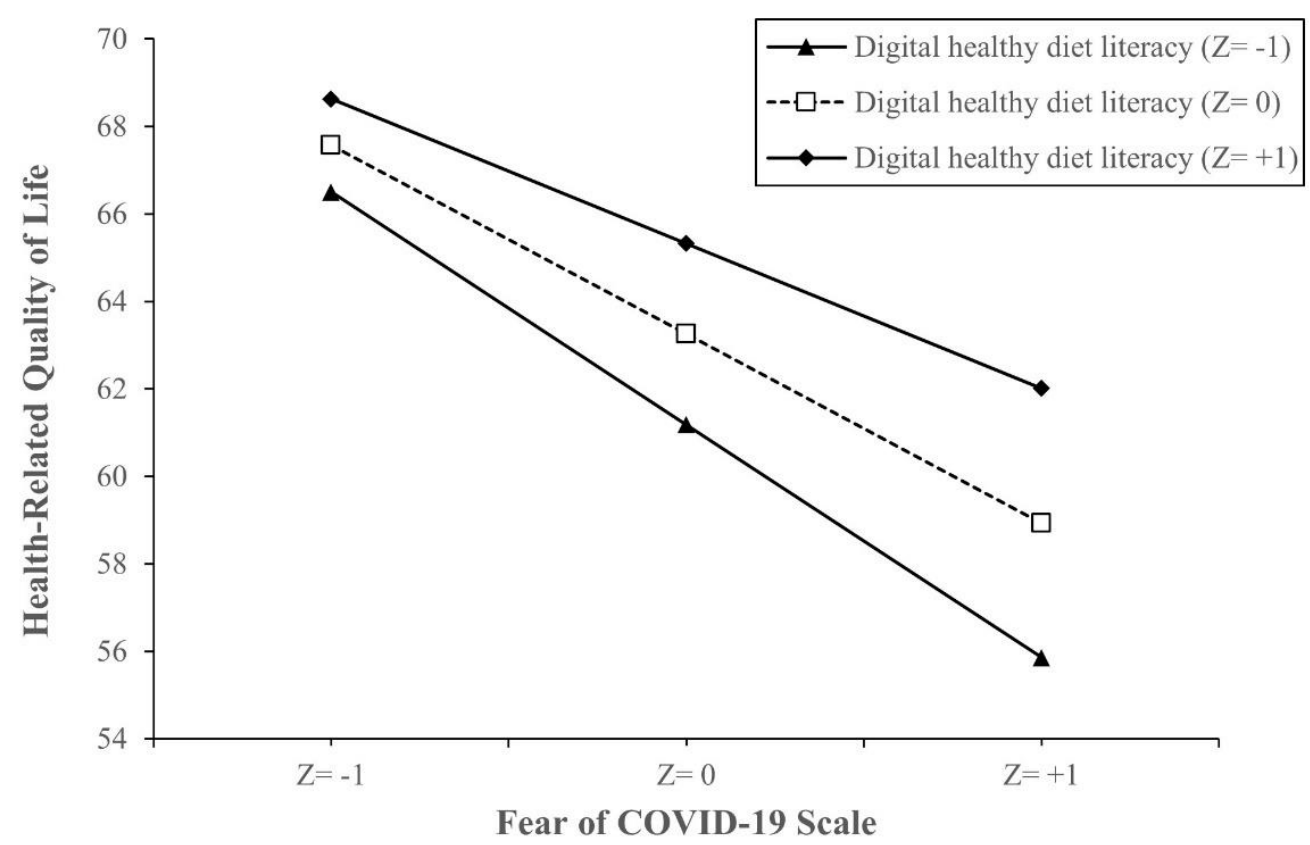

Figure 2. Simple slope plot of interaction between digital healthy diet literacy and fear of COVID-19 on health-related quality of life $(\mathrm{N}=4348)$. Note: $\mathrm{Z}=-1$, one SD below the mean; $\mathrm{Z}=0$, the mean; $\mathrm{Z}$ $=+1,1 \mathrm{SD}$ above the mean. Z-score is the number of $\mathrm{SD}$ above or below the mean. Abbreviations: $\mathrm{SD}$, standard deviation.

\section{Discussion}

In this study, people with higher FCoV-19S scores had lower HRQoL scores. This finding was consistent with a previous study among the general population in China [45]. Several reasons could also explain this association. First, fears of COVID-19 transmission can cause mental health problems such as anxiety, depression, or even suicide [46,47], which may negatively affect the HRQoL [48]. Secondly, patients may be delayed access to healthcare systems due to fears of disease infection, especially in patients with chronic diseases [9]. Besides, during the COVID-19 crisis, the fear of COVID-19 may cause sedentary behaviors and negative lifestyle changes such as unhealthy diets, inactive physical activities, or substance abuse $[37,49]$. All of these factors may worsen physical health and well-being [50]. Therefore, it is essential to develop timely public health strategies to mitigate the concerns and fears related to COVID-19, which may improve the quality of life among patients.

In the current study, higher scores of health literacy and eHealth literacy were associated with higher HRQoL scores. These findings were similar to results from previous studies $[19,51]$. Besides, higher health literacy and eHealth literacy were also positively associated with a healthy diet and physical exercise [18,52], which may further improve well-being.

Our study showed that eHealth literacy could alleviate the adverse effect of FCoV-19S on health-related quality of life, whereas health literacy was not effective in the model of interaction analyses. These results might be explained by the fact that during the COVID-19 pandemic, people spent more time on the Internet and social media for communicating and finding information [49]. They also faced a wide range of disinformation and misinformation about COVID-19, causing confusion and fears $[53,54]$. Therefore, people with a high degree of eHealth literacy may better find reliable sources and appraise online health information, thereby reducing the FCoV-19S and improving the HRQoL [17].

In addition, as social media and news websites have rapidly become essential tools for communicating and disseminating information for the community [12,13], the critical role of eHealth literacy has been highlighted during the pandemic. That is why health literacy could enhance the quality of life in our study, but it could not mitigate the harmful effect of 
FCoV-19S on HRQoL. However, the role of health literacy has still been crucial both before and during the pandemic [55].

Therefore, timely and comprehensive interventions need to be developed to boost health literacy and eHealth literacy for communities, which would further improve the quality of life amid the pandemic, especially in patients with fears of COVID-19. A previous study showed that a higher fear of COVID-19 score was associated with a lower health literacy [37]. Thus, to improve health literacy and e-health literacy, governments and health organizations need to inform the community through mass media with timely, accurate, transparent, and plain information about the COVID-19 epidemic, such as COVID-19 symptoms, means of transmission, and effective prevention measures. Another critical approach is providing accessible, reliable, and trusted health information sources (e.g., credible websites or forums) that assist individuals in enhancing their beneficial health knowledge and in avoiding COVID-19-related misinformation during the pandemic. As smart devices are becoming more popular, technological solutions also need to be applied (e.g., patient portals, telemedicine, and health-related mobile applications) to help healthcare providers connect and consult more effectively with patients, which may make a significant contribution to enhancing patient's health literacy [56].

We found that people with higher DDL scores had higher HRQoL scores. In the interaction model, a higher DDL score significantly weakened the negative impact of FCoV-19S on HRQoL. These findings could be explained by people with better DDL having a higher likelihood of adherence to healthier eating behaviors during the pandemic [30]. Another study conducted among the adult population in the Netherlands also indicated that higher food literacy was positively associated with a healthy diet [57]. Meanwhile, previous studies have suggested that the adherence to healthy dietary patterns could enhance the health-related quality of life [58-61]. Balanced and nutritious diets help to improve health status, reduce the risk of chronic diseases [62,63], and potentially reduce the risk of complications and severe conditions of COVID-19 [25]. Better diet quality was found to be associated with a lower risk of depression [26,64]. Thus, better DDL may help patients with COVID-19-related fears have a better dietary intake, thereby strengthening their physical and mental health, and their quality of life may gradually improve. This suggests that DDL is a critical issue that should be highlighted for intervention promotion to improve the HRQoL, especially in the digital age with too many inaccurate information sources on the internet.

Our study had some limitations. First, as the survey was conducted amid the pandemic, both research assistants and studied respondents were highly vulnerable to COVID19 infection. Thus, all interviewers were requested to adhere to the national guidelines about infection prevention throughout the data collection procedure. Fortunately, no new confirmed cases were reported during the period of data collection [65]. Second, a causal relationship could not be drawn from the cross-sectional study. Thirdly, our discussion focused on explaining the relationship between DDL and HRQoL through improving healthy eating behaviors. However, the relationship between DDL and healthy eating behaviors could be modified by several factors (e.g., income, occupation, social status, and food insecurity). Therefore, future studies need to confirm our findings. Finally, the findings should be generalized to the outpatient population with caution due to the convenient sampling method. However, as our study was conducted with a relatively large sample size, the results may produce timely evidence for developing public health interventions to improve the health-related quality of life, especially in those with fears of COVID-19 during the pandemic.

\section{Conclusions}

In this study, the fear of COVID-19 was negatively associated with the health-related quality of life. In contrast, health literacy, eHealth literacy, and digital healthy diet literacy were positively associated with the health-related quality of life. eHealth literacy and digital healthy diet literacy can help to mitigate the negative effects of the fear of COVID-19 on the 
health-related quality of life. Strategical public health interventions should be promoted to improve eHealth literacy and digital healthy diet literacy, which would further enhance the health-related quality of life, especially among patients with fears of COVID-19.

Supplementary Materials: The following are available online at https://www.mdpi.com/article/ 10.3390/ijerph18094929/s1, Table S1. Confounders associated with health-related quality of life among outpatients $(\mathrm{N}=4348)$. Table S2. Spearman's correlations (rho) among the studied variables $(\mathrm{N}=4348)$. Table S3. Pearson's correlations (rho) among fears of COVID-19, digital healthy diet literacy, eHealth literacy, and health literacy $(\mathrm{N}=4348)$.

Author Contributions: Conceptualization, M.H.N., T.T.M.P., K.T.N., Y.H.N., T.V.T., B.N.D., H.K.D., H.C.N., N.T.D., T.H.H., D.T.P., K.M.P., L.V.P., P.B.N., H.T.T.N., T.V.D. (Thinh V. Do), D.T.H., H.Q.N., H.T.M.N., M.V.T., T.T.T.M., N.P.T.N., A.L.T., T.T.P.N., K.T.N., C.-H.B. and T.V.D. (Tuyen Van Duong); methodology, M.H.N., T.T.M.P., K.T.N., Y.H.N., T.V.T., B.N.D., H.K.D., H.C.N., N.T.D., T.H.H., D.T.P., K.M.P., L.V.P., P.B.N., H.T.T.N., T.V.D. (Thinh V. Do), D.T.H., H.Q.N., H.T.M.N., M.V.T., T.T.T.M., N.P.T.N., A.L.T., T.T.P.N., K.T.N., C.-H.B. and T.V.D. (Tuyen Van Duong); software, M.H.N., C.-H.B. and T.V.D. (Tuyen Van Duong); validation, M.H.N., T.T.M.P., K.T.N., Y.H.N., T.V.T., B.N.D., H.K.D., H.C.N., N.T.D., T.H.H., D.T.P., K.M.P., L.V.P., P.B.N., H.T.T.N., T.V.D. (Thinh V. Do), D.T.H., H.Q.N., H.T.M.N., M.V.T., T.T.T.M., N.P.T.N., A.L.T., T.T.P.N., K.T.N., C.-H.B. and T.V.D. (Tuyen Van Duong); formal analysis, M.H.N., C.-H.B. and T.V.D. (Tuyen Van Duong); investigation, M.H.N., T.T.M.P., K.T.N., Y.H.N., T.V.T., B.N.D., H.K.D., H.C.N., N.T.D., T.H.H., D.T.P., K.M.P., L.V.P., P.B.N., H.T.T.N., T.V.D. (Thinh V. Do), D.T.H., H.Q.N., H.T.M.N., M.V.T., T.T.T.M., N.P.T.N., A.L.T., T.T.P.N., K.T.N., C.-H.B. and T.V.D. (Tuyen Van Duong); data curation, M.H.N., T.T.M.P., K.T.N., Y.H.N., T.V.T., B.N.D., H.K.D., H.C.N., N.T.D., T.H.H., D.T.P., K.M.P., L.V.P., P.B.N., H.T.T.N., T.V.D. (Thinh V. Do), D.T.H., H.Q.N., H.T.M.N., M.V.T., T.T.T.M., N.P.T.N., A.L.T., T.T.P.N., K.T.N., and T.V.D. (Tuyen Van Duong); writing—original draft preparation, M.H.N., C.-H.B. and T.V.D. (Tuyen Van Duong); writing-review and editing, M.H.N., T.T.M.P., K.T.N., Y.H.N., T.V.T., B.N.D., H.K.D., H.C.N., N.T.D., T.H.H., D.T.P., K.M.P., L.V.P., P.B.N., H.T.T.N., T.V.D. (Thinh V. Do), D.T.H., H.Q.N., H.T.M.N., M.V.T., T.T.T.M., N.P.T.N., A.L.T., T.T.P.N., K.T.N., C.-H.B. and T.V.D. (Tuyen Van Duong); supervision, C.-H.B. and T.V.D. (Tuyen Van Duong); project admin-istration, M.H.N., T.T.P.N., T.T.M.P. and T.V.D. (Tuyen Van Duong); funding acquisition, C.-H.B. and T.V.D. (Tuyen Van Duong). All authors have read and agreed to the published version of the manuscript.

Funding: This study was supported by Taipei Medical University (108-6202-008-112; 108-3805-022400) and the Ministry of Science and Technology of Taiwan (MOST 107-2314-B-038-072-MY3).

Institutional Review Board Statement: The study was reviewed and approved by the Institutional Ethical Review Committee of Hanoi University of Public Health, Vietnam (IRB No. 133/2020/ YTCC-HD3).

Informed Consent Statement: Informed consent was obtained from all subjects involved in the study.

Data Availability Statement: Data will be available on the reasonable request from the corresponding author.

Acknowledgments: The authors would like to thank the medical staff and medical students who helped with data collection. We also appreciate and acknowledge the participation of OPD visitors from the selected hospitals.

Conflicts of Interest: The authors declare no conflict of interest.

\author{
Abbreviations \\ B \\ DDL \\ eHEALS \\ FCoV-19S \\ HL \\ HLS-SF12 \\ HRQoL \\ OPD \\ S-COVID-19-S
}




\section{References}

1. Phelan, A.L.; Katz, R.; Gostin, L.O. The Novel Coronavirus Originating in Wuhan, China: Challenges for Global Health Governance. JAMA 2020, 323, 709-710. [CrossRef] [PubMed]

2. Nicola, M.; Alsafi, Z.; Sohrabi, C.; Kerwan, A.; Al-Jabir, A.; Iosifidis, C.; Agha, M.; Agha, R. The socio-economic implications of the coronavirus pandemic (COVID-19): A review. Int. J. Surg. 2020, 78, 185-193. [CrossRef] [PubMed]

3. Greenberg, N.; Docherty, M.; Gnanapragasam, S.; Wessely, S. Managing mental health challenges faced by healthcare workers during covid-19 pandemic. BMJ 2020, 368, m1211. [CrossRef] [PubMed]

4. World Health Organisation. Coronavirus disease (COVID-19) Weekly Epidemiological Update and Weekly Operational Update. Available online: https://www.who.int/emergencies/diseases/novel-coronavirus-2019/situation-reports (accessed on 15 September 2020).

5. Nazir, M.; Hussain, I.; Tian, J.; Akram, S.; Mangenda Tshiaba, S.; Mushtaq, S.; Shad, M.A. A Multidimensional Model of Public Health Approaches Against COVID-19. Int. J. Environ. Res. Public Health 2020, 17, 3780. [CrossRef] [PubMed]

6. Wei, K.; Nyunt, M.S.Z.; Gao, Q.; Wee, S.L.; Ng, T.P. Long-term changes in nutritional status are associated with functional and mortality outcomes among community-living older adults. Nutrition 2019, 66, 180-186. [CrossRef]

7. Holmes, E.A.; O'Connor, R.C.; Perry, V.H.; Tracey, I.; Wessely, S.; Arseneault, L.; Ballard, C.; Christensen, H.; Cohen Silver, R.; Everall, I.; et al. Multidisciplinary research priorities for the COVID-19 pandemic: A call for action for mental health science. Lancet Psychiatry 2020, 7, 547-560. [CrossRef]

8. Goyal, K.; Chauhan, P.; Chhikara, K.; Gupta, P.; Singh, M.P. Fear of COVID 2019: First suicidal case in India! Asian J. Psychiatr. 2020, 49, 101989. [CrossRef]

9. Lazzerini, M.; Barbi, E.; Apicella, A.; Marchetti, F.; Cardinale, F.; Trobia, G. Delayed access or provision of care in Italy resulting from fear of COVID-19. Lancet Child Adolesc Health 2020, 4, e10-e11. [CrossRef]

10. World Health Organisation. COVID-19: Vulnerable and High Risk Groups. Available online: https://www.who.int/ westernpacific/emergencies/covid-19/information/high-risk-groups (accessed on 8 May 2020).

11. Yang, J.; Zheng, Y.; Gou, X.; Pu, K.; Chen, Z.F.; Guo, Q.H.; Ji, R.; Wang, H.J.; Wang, Y.P.; Zhou, Y.N. Prevalence of comorbidities and its effects in patients infected with SARS-CoV-2: A systematic review and meta-analysis. Int. J. Infect. Dis. 2020, 94, 91-95. [CrossRef]

12. Tsao, S.F.; Chen, H.; Tisseverasinghe, T.; Yang, Y.; Li, L.; Butt, Z.A. What social media told us in the time of COVID-19: A scoping review. Lancet Digit Health 2021, 3, e175-e194. [CrossRef]

13. Liao, Q.; Yuan, J.; Dong, M.; Yang, L.; Fielding, R.; Lam, W.W.T. Public Engagement and Government Responsiveness in the Communications About COVID-19 During the Early Epidemic Stage in China: Infodemiology Study on Social Media Data. J. Med. Internet Res. 2020, 22, e18796. [CrossRef]

14. Bao, Y.; Sun, Y.; Meng, S.; Shi, J.; Lu, L. 2019-nCoV epidemic: Address mental health care to empower society. Lancet 2020, 395, e37-e38. [CrossRef]

15. Norman, C.D.; Skinner, H.A. eHealth Literacy: Essential Skills for Consumer Health in a Networked World. J. Med. Internet Res. 2006, 8, e9. [CrossRef]

16. Diviani, N.; van den Putte, B.; Giani, S.; van Weert, J.C. Low health literacy and evaluation of online health information: A systematic review of the literature. J. Med. Internet Res. 2015, 17, e112. [CrossRef] [PubMed]

17. Chong, Y.Y.; Cheng, H.Y.; Chan, H.Y.L.; Chien, W.T.; Wong, S.Y.S. COVID-19 pandemic, infodemic and the role of eHealth literacy. Int. J. Nurs. Stud. 2020, 108, 103644. [CrossRef]

18. Do, B.N.; Tran, T.V.; Phan, D.T.; Nguyen, H.C.; Nguyen, T.T.P.; Nguyen, H.C.; Ha, T.H.; Dao, H.K.; Trinh, M.V.; Do, T.V.; et al. Health Literacy, eHealth Literacy, Adherence to Infection Prevention and Control Procedures, Lifestyle Changes, and Suspected COVID-19 Symptoms Among Health Care Workers During Lockdown: Online Survey. J. Med. Internet Res. 2020, 22, e22894. [CrossRef] [PubMed]

19. Greenhalgh, T. Health literacy: Towards system level solutions. BMJ 2015, 350, h1026. [CrossRef]

20. Gasmi, A.; Noor, S.; Tippairote, T.; Dadar, M.; Menzel, A.; Bjørklund, G. Individual risk management strategy and potential therapeutic options for the COVID-19 pandemic. Clin. Immunol. 2020, 215, 108409. [CrossRef] [PubMed]

21. Zhang, L.; Liu, Y. Potential interventions for novel coronavirus in China: A systematic review. J. Med. Virol. 2020, 92, 479-490. [CrossRef] [PubMed]

22. Kakodkar, P.; Kaka, N.; Baig, M.N. A Comprehensive Literature Review on the Clinical Presentation, and Management of the Pandemic Coronavirus Disease 2019 (COVID-19). Cureus 2020, 12, e7560. [CrossRef] [PubMed]

23. Morais, A.H.A.; Aquino, J.S.; da Silva-Maia, J.K.; Vale, S.H.L.; Maciel, B.L.L.; Passos, T.S. Nutritional status, diet and viral respiratory infections: Perspectives for severe acute respiratory syndrome coronavirus 2. Br. J. Nutr. 2020, 1-12. [CrossRef] [PubMed]

24. Jayedi, A.; Soltani, S.; Abdolshahi, A.; Shab-Bidar, S. Healthy and unhealthy dietary patterns and the risk of chronic disease: An umbrella review of meta-analyses of prospective cohort studies. Br. J. Nutr. 2020, 124, 1133-1144. [CrossRef]

25. Butler, M.J.; Barrientos, R.M. The impact of nutrition on COVID-19 susceptibility and long-term consequences. Brain. Behav. Immun. 2020, 87, 53-54. [CrossRef] [PubMed] 
26. Pham, K.M.; Pham, L.V.; Phan, D.T.; Tran, T.V.; Nguyen, H.C.; Nguyen, M.H.; Nguyen, H.C.; Ha, T.H.; Dao, H.K.; Nguyen, P.B. Healthy Dietary Intake Behavior Potentially Modifies the Negative Effect of COVID-19 Lockdown on Depression: A Hospital and Health Center Survey. Front. Nutr. 2020, 7, 230. [CrossRef]

27. Pike, K.M.; Dunne, P.E. The rise of eating disorders in Asia: A review. J. Eat. Disord. 2015, 3, 33. [CrossRef] [PubMed]

28. GBD 2017 Diet Collaborators. Health effects of dietary risks in 195 countries, 1990-2017: A systematic analysis for the Global Burden of Disease Study 2017. Lancet 2019, 393, 1958-1972. [CrossRef]

29. Nguyen, T.T.; Hoang, M.V. Non-communicable diseases, food and nutrition in Vietnam from 1975 to 2015 : The burden and national response. Asia Pac. J. Clin. Nutr. 2018, 27, 19-28. [CrossRef]

30. Duong, T.V.; Pham, K.M.; Do, B.N.; Kim, G.B.; Dam, H.T.B.; Le, V.T.; Nguyen, T.T.P.; Nguyen, H.T.; Nguyen, T.T.; Le, T.T.; et al. Digital Healthy Diet Literacy and Self-Perceived Eating Behavior Change during COVID-19 Pandemic among Undergraduate Nursing and Medical Students: A Rapid Online Survey. Int. J. Environ. Res. Public Health 2020, 17, 7185. [CrossRef] [PubMed]

31. World Health Organization. Novel Coronavirus (2019-nCoV) Technical Guidance. Available online: https:/ /www.who.int/ emergencies/diseases/novel-coronavirus-2019/technicalguidance (accessed on 15 April 2020).

32. Hays, R.D.; Morales, L.S. The RAND-36 measure of health-related quality of life. Ann. Med. 2001, 33, 350-357. [CrossRef]

33. Tran, T.V.; Nguyen, H.C.; Pham, L.V.; Nguyen, M.H.; Nguyen, H.C.; Ha, T.H.; Phan, D.T.; Dao, H.K.; Nguyen, P.B.; Trinh, M.V.; et al. Impacts and interactions of COVID-19 response involvement, health-related behaviours, health literacy on anxiety, depression and health-related quality of life among healthcare workers: A cross-sectional study. BMJ Open 2020, 10, e041394. [CrossRef]

34. Nguyen, H.C.; Nguyen, M.H.; Do, B.N.; Tran, C.Q.; Nguyen, T.T.P.; Pham, K.M.; Pham, L.V.; Tran, K.V.; Duong, T.T.; Tran, T.V.; et al. People with Suspected COVID-19 Symptoms Were More Likely Depressed and Had Lower Health-Related Quality of Life: The Potential Benefit of Health Literacy. J. Clin. Med. 2020, 9, 965. [CrossRef]

35. Hays, R.D.; Sherbourne, C.D.; Mazel, R.M. The rand 36-item health survey 1.0. Health Econ. 1993, 2, 217-227. [CrossRef] [PubMed]

36. Ahorsu, D.K.; Lin, C.Y.; Imani, V.; Saffari, M.; Griffiths, M.D.; Pakpour, A.H. The Fear of COVID-19 Scale: Development and Initial Validation. Int. J. Ment. Health Addict. 2020, 1-9. [CrossRef] [PubMed]

37. Nguyen, H.T.; Do, B.N.; Pham, K.M.; Kim, G.B.; Dam, H.T.B.; Nguyen, T.T.; Nguyen, T.T.P.; Nguyen, Y.H.; Sørensen, K.; Pleasant, A.; et al. Fear of COVID-19 Scale-Associations of Its Scores with Health Literacy and Health-Related Behaviors among Medical Students. Int. J. Environ. Res. Public Health 2020, 17, 4164. [CrossRef] [PubMed]

38. Duong, T.V.; Aringazina, A.; Kayupova, G.; Pham, T.V.; Pham, K.M.; Truong, T.Q.; Nguyen, K.T.; Oo, W.M.; Su, T.T.; Majid, H.A. Development and validation of a new short-form health literacy instrument (HLS-SF12) for the general public in six Asian countries. Health Lit. Res. Pract. 2019, 3, e91-e102. [CrossRef]

39. Duong, T.V.; Nguyen, T.T.P.; Pham, K.M.; Nguyen, K.T.; Giap, M.H.; Tran, T.D.X.; Nguyen, C.X.; Yang, S.H.; Su, C.T. Validation of the Short-Form Health Literacy Questionnaire (HLS-SF12) and Its Determinants among People Living in Rural Areas in Vietnam. Int. J. Environ. Res. Public Health 2019, 16, 3346. [CrossRef] [PubMed]

40. Nguyen, T.T.; Le, N.T.; Nguyen, M.H.; Pham, L.V.; Do, B.N.; Nguyen, H.C.; Nguyen, H.C.; Ha, T.H.; Dao, H.K.; Nguyen, P.B.; et al. Health Literacy and Preventive Behaviors Modify the Association between Pre-Existing Health Conditions and Suspected COVID-19 Symptoms: A Multi-Institutional Survey. Int. J. Environ. Res. Public Health 2020, 17, 8598. [CrossRef]

41. Do, B.N.; Nguyen, P.A.; Pham, K.M.; Nguyen, H.C.; Nguyen, M.H.; Tran, C.Q.; Nguyen, T.T.P.; Tran, T.V.; Pham, L.V.; Tran, K.V.; et al. Determinants of Health Literacy and Its Associations With Health-Related Behaviors, Depression Among the Older People With and Without Suspected COVID-19 Symptoms: A Multi-Institutional Study. Front. Public Health 2020, 8, 581746. [CrossRef]

42. BMJ Editorial Team. Overview of novel coronavirus (2019-nCoV). Available online: https://bestpractice.bmj.com/topics/en-gb/ 3000165 (accessed on 10 March 2020).

43. Charlson, M.E.; Pompei, P.; Ales, K.L.; MacKenzie, C.R. A new method of classifying prognostic comorbidity in longitudinal studies: Development and validation. J. Chronic Dis. 1987, 40, 373-383. [CrossRef]

44. World Health Organisation. Coronavirus Disease (COVID-19): Advice for the Public. Available online: https://www.who.int/ emergencies/diseases/novel-coronavirus-2019/advice-for-public (accessed on 15 December 2020).

45. Ping, W.; Zheng, J.; Niu, X.; Guo, C.; Zhang, J.; Yang, H.; Shi, Y. Evaluation of health-related quality of life using EQ-5D in China during the COVID-19 pandemic. PLoS ONE 2020, 15, e0234850. [CrossRef]

46. Fitzpatrick, K.M.; Harris, C.; Drawve, G. Living in the midst of fear: Depressive symptomatology among US adults during the COVID-19 pandemic. Depress. Anxiety 2020, 37, 957-964. [CrossRef]

47. Mamun, M.A.; Griffiths, M.D. First COVID-19 suicide case in Bangladesh due to fear of COVID-19 and xenophobia: Possible suicide prevention strategies. Asian J. Psychiatr. 2020, 51, 102073. [CrossRef]

48. Ma, Y.F.; Li, W.; Deng, H.B.; Wang, L.; Wang, Y.; Wang, P.H.; Bo, H.X.; Cao, J.; Wang, Y.; Zhu, L.Y.; et al. Prevalence of depression and its association with quality of life in clinically stable patients with COVID-19. J. Affect. Disord. 2020, 275, 145-148. [CrossRef] [PubMed]

49. Górnicka, M.; Drywień, M.E.; Zielinska, M.A.; Hamułka, J. Dietary and Lifestyle Changes During COVID-19 and the Subsequent Lockdowns among Polish Adults: A Cross-Sectional Online Survey PLifeCOVID-19 Study. Nutrients 2020, 12, 2324. [CrossRef] [PubMed] 
50. Hu, Z.; Lin, X.; Chiwanda Kaminga, A.; Xu, H. Impact of the COVID-19 Epidemic on Lifestyle Behaviors and Their Association With Subjective Well-Being Among the General Population in Mainland China: Cross-Sectional Study. J. Med. Internet Res. 2020, 22, e21176. [CrossRef] [PubMed]

51. Watson, R. Europeans with poor "health literacy" are heavy users of health services. BMJ 2011, 343, d7741. [CrossRef]

52. Britt, R.K.; Collins, W.B.; Wilson, K.; Linnemeier, G.; Englebert, A.M. eHealth Literacy and Health Behaviors Affecting Modern College Students: A Pilot Study of Issues Identified by the American College Health Association. J. Med. Internet Res. 2017, 19, e392. [CrossRef]

53. Ahmad, A.R.; Murad, H.R. The Impact of Social Media on Panic During the COVID-19 Pandemic in Iraqi Kurdistan: Online Questionnaire Study. J. Med. Internet Res. 2020, 22, e19556. [CrossRef]

54. Shimizu, K. 2019-nCoV, fake news, and racism. Lancet 2020, 395, 685-686. [CrossRef]

55. Sentell, T.; Vamos, S.; Okan, O. Interdisciplinary Perspectives on Health Literacy Research Around the World: More Important Than Ever in a Time of COVID-19. Int. J. Environ. Res. Public Health 2020, 17, 3010. [CrossRef]

56. ACP Decisions. Four Simple Strategies for Improving Your Patients' Health Literacy. Available online: https: / acpdecisions.org/ four-simple-strategies-for-improving-your-patients-health-literacy/ (accessed on 15 March 2021).

57. Poelman, M.P.; Dijkstra, S.C.; Sponselee, H.; Kamphuis, C.B.M.; Battjes-Fries, M.C.E.; Gillebaart, M.; Seidell, J.C. Towards the measurement of food literacy with respect to healthy eating: The development and validation of the self perceived food literacy scale among an adult sample in the Netherlands. Int. J. Behav. Nutr. Phys. Act. 2018, 15, 54. [CrossRef] [PubMed]

58. Bonaccio, M.; Di Castelnuovo, A.; Bonanni, A.; Costanzo, S.; De Lucia, F.; Pounis, G.; Zito, F.; Donati, M.B.; de Gaetano, G.; Iacoviello, L. Adherence to a Mediterranean diet is associated with a better health-related quality of life: A possible role of high dietary antioxidant content. BMJ Open 2013, 3. [CrossRef] [PubMed]

59. Xu, F.; Cohen, S.A.; Lofgren, I.E.; Greene, G.W.; Delmonico, M.J.; Greaney, M.L. Relationship between Diet Quality, Physical Activity and Health-Related Quality of Life in Older Adults: Findings from 2007-2014 National Health and Nutrition Examination Survey. J. Nutr. Health Aging 2018, 22, 1072-1079. [CrossRef] [PubMed]

60. Wu, X.Y.; Zhuang, L.H.; Li, W.; Guo, H.W.; Zhang, J.H.; Zhao, Y.K.; Hu, J.W.; Gao, Q.Q.; Luo, S.; Ohinmaa, A.; et al. The influence of diet quality and dietary behavior on health-related quality of life in the general population of children and adolescents: A systematic review and meta-analysis. Qual. Life Res. 2019, 28, 1989-2015. [CrossRef] [PubMed]

61. Milte, C.M.; Thorpe, M.G.; Crawford, D.; Ball, K.; McNaughton, S.A. Associations of diet quality with health-related quality of life in older Australian men and women. Exp. Gerontol. 2015, 64, 8-16. [CrossRef]

62. McCullough, M.L.; Feskanich, D.; Stampfer, M.J.; Giovannucci, E.L.; Rimm, E.B.; Hu, F.B.; Spiegelman, D.; Hunter, D.J.; Colditz, G.A.; Willett, W.C. Diet quality and major chronic disease risk in men and women: Moving toward improved dietary guidance. Am. J. Clin. Nutr. 2002, 76, 1261-1271. [CrossRef] [PubMed]

63. Schulze, M.B.; Martínez-González, M.A.; Fung, T.T.; Lichtenstein, A.H.; Forouhi, N.G. Food based dietary patterns and chronic disease prevention. BMJ 2018, 361, k2396. [CrossRef] [PubMed]

64. Molendijk, M.; Molero, P.; Ortuño Sánchez-Pedreño, F.; Van der Does, W.; Angel Martínez-González, M. Diet quality and depression risk: A systematic review and dose-response meta-analysis of prospective studies. J. Affect. Disord. 2018, 226, 346-354. [CrossRef]

65. Vietnam Ministry of Health. Coronavirus Disease (COVID-19) Outbreak in Vietnam. Available online: https://ncov.moh.gov.vn/ (accessed on 7 June 2020). 